\title{
Clinical characteristics and risk factors of polymicrobial Staphylococcus aureus bloodstream infections
}

Cheng Zheng ${ }^{1,2 \dagger}$, Shufang Zhang ${ }^{3 \dagger}$, Qingqing Chen ${ }^{4 \dagger}$, Li Zhong ${ }^{1,5}$, Tiancha Huang ${ }^{1}$, Xijiang Zhang ${ }^{2}$, Kai Zhang ${ }^{1}$, Hongwei Zhou', Jiachang Cai ${ }^{6}$, Linlin Du', Changming Wang ${ }^{2}$, Wei Cui ${ }^{1}$ and Gensheng Zhang ${ }^{1 *}$ (D)

\begin{abstract}
Background: Although Staphylococcus aureus bloodstream infections (SA-BSI) are a common and important infection, polymicrobial SA-BSI are infrequently reported. The aim of this study was to investigate the clinical characteristics and risk factors of polymicrobial SA-BSI in comparison with monomicrobial SA-BSI.
\end{abstract}

Methods: A single-center retrospective observational study was performed between Jan 1, 2013, and Dec 31, 2018 at a tertiary hospital. All patients with SA-BSI were enrolled, and their clinical data were gathered by reviewing electronic medical records.

Results: A total of 349 patients with SA-BSI were enrolled including 54 cases (15.5\%) with polymicrobial SA-BSI. In multivariable analysis, burn injury (adjusted odds ratio [OR], 7.04; 95\% confidence interval [Cl], 1.71-28.94), need of blood transfusion (aOR, 2.72; 95\% Cl, 1.14-6.50), use of mechanical ventilation (aOR, 3.11; 95\% Cl, 1.16-8.30), the length of prior hospital stay $(\mathrm{aOR}, 1.02 ; 95 \% \mathrm{Cl}, 1.00-1.03)$, and pneumonia as primary site of infection (aOR, 4.22; $95 \% \mathrm{Cl}, 1.69-10.51$ ) were independent factors of polymicrobial SA-BSI. In comparison with monomicrobial SA-BSI, patients with polymicrobial SA-BSI had longer length of ICU stay [median days, $23(6.25,49.25)$ vs. $0(0,12), p<0.01$ ] and hospital stay [median days, 50(21.75,85.75) vs. 28(15,49), $p<0.01$ ], and showed a higher 28-day mortality $(29.6 \%$ vs. $15.3 \%, p=0.01)$.

Conclusions: Burn injury, blood transfusion, mechanical ventilation, the length of prior hospital stay, and pneumonia as a primary site of infection are independent risk factors for polymicrobial SA-BSI. In addition, patients with polymicrobial SA-BSI might have worse outcomes compared with monomicrobial SA-BSI.

Keywords: Staphylococcus aureus, Bacteremia, Polymicrobial Staphylococcus aureus bloodstream infections, Risk factors, Mortality

\footnotetext{
* Correspondence: genshengzhang@zju.edu.cn

${ }^{+}$Cheng Zheng, Shufang Zhang and Qingqing Chen contributed equally to this work.

${ }^{1}$ Department of Critical Care Medicine, Second Affiliated Hospital, Zhejiang University School of Medicine, Hangzhou 310009, China

Full list of author information is available at the end of the article
}

(c) The Author(s). 2020 Open Access This article is licensed under a Creative Commons Attribution 4.0 International License, which permits use, sharing, adaptation, distribution and reproduction in any medium or format, as long as you give appropriate credit to the original author(s) and the source, provide a link to the Creative Commons licence, and indicate if changes were made. The images or other third party material in this article are included in the article's Creative Commons licence, unless indicated otherwise in a credit line to the material. If material is not included in the article's Creative Commons licence and your intended use is not permitted by statutory regulation or exceeds the permitted use, you will need to obtain permission directly from the copyright holder. To view a copy of this licence, visit http://creativecommons.org/licenses/by/4.0/ The Creative Commons Public Domain Dedication waiver (http://creativecommons.org/publicdomain/zero/1.0/) applies to the data made available in this article, unless otherwise stated in a credit line to the data. 


\section{background}

Due to their potentially serious consequences, bloodstream infections (BSI) are a growing worldwide concern [1]. BSI can be caused by a wide variety of microorganisms, and the most common organisms were Coagulasenegative staphylococci (CNS), Staphylococcus aureus ( $S$. aureus), Enterococci, and Candida species [2]. S. aureus is the second most common cause of BSI, which also serves as the most important cause of BSI-associated death $[3,4]$. Most of BSI are monomicrobial, but the trend of polymicrobial BSI is rising with a range of $6 \%$ $34 \%$ among BSI $[2,5,6]$. Polymicrobial BSI is generally associated with a higher acute physiology and chronic health evaluation (APACHE) II scores, prolonged ICU and hospital stay, and a more severe prognosis than monomicrobial BSI in adults [5-9]. In these previous studies [5-11], some limitations also existed as follows: (1) The clinical significance and outcomes of polymicrobial versus monomicrobial BSI were indeed investigated, but few reports focused on a specific pathogen. Thus, the specific clinical features and outcomes between polymicrobial SA-BSI and monomicrobial SA-BSI are still largely unknown. (2) In a previous study [10], patients with polymicrobial SA-BSI often had a biliary source and had a worse prognosis, and independent risk factors for polymicrobial SA-BSI included neutropenia, biliary tract catheters, and intra-abdominal infection. A bias was also pointed out that a high proportion of biliary tract diseases (7\%) was observed in their institution [10]. (3) Another study illustrated that SA-BSI was usually monomicrobial, and soft tissue was the most common source [11]. Unfortunately, this study did not investigate clinical characteristics and the risk factors for polymicrobial SA-BSI. (4) However, these two studies [10, 11] focused on Korean and American population respectively, and there were no studies focused on Chinese population at present. Thus, the clinical outcomes between polymicrobial SA-BSI and monomicrobial SA-BSI are still controversial. Herein, we conducted the retrospective study on polymicrobial SA-BSI to provide more information of the clinical characteristics and risk factors of polymicrobial SA-BSI.

\section{Materials and methods}

\section{Patients and study design}

This single-center retrospective cohort study was conducted from January 2013 to December 2018 in the Second Affiliated Hospital, Zhejiang University School of Medicine, a 3200-bed tertiary-level healthcare facility in Hangzhou, China. The present study received human research ethics approval (No. 2019-194) from the Ethics Committee of the Second Affiliated Hospital, Zhejiang University School of Medicine. Due to the retrospective nature of the study, the Ethics Committee determined that no patient consent was required. In addition, a statement of permission from patients for submission was not required as the study did not include any personal information.

If any microorganisms besides $S$. aureus were found in the same blood culture, the cases were retained. If only $S$. aureus was found in multiple blood cultures of the same patient, the patients were included only one time when they happened with SA-BSI at the first time. Exclusion criteria were as follows: a) Age $<18$ years old; b) $S$. aureus considered as nonpathogenic bacterium (In bilateral double bottles blood culture, S. aureus was only cultured in one bottle, and the culture time was more than 48 h); c) Cases data were incomplete or missing; d) Loss to follow-up. Common skin contaminant organisms (eg, Bacillus spp., Corynebacterium spp., Micrococcus spp., Streptococci, Lactobacillus spp. and CNS) were considered pathogens only when they were present in two or more consecutive blood cultures from separate blood draws. Thus, a total of 1174 blood culture specimens containing $S$. aureus were initially included, and 349 cases were finally recruited with 54 cases of polymicrobial SA-BSI and 295 cases of monomicrobial SA-BSI (Fig. 1).

\section{Data collection}

The patients' data were collected by reviewing electronic medical records. We recorded demographic data including age and gender, the clinical data including underlying diseases, Sequential Organ Failure Assessment (SOFA) score, Pitt bacteremia score, Charlson Comorbidity Index (CCI) score, APACHE II score in the first $24 \mathrm{~h}$ following the onset of BSI, the hospitalization wards, nosocomial infection or not, previous exposures (prior hospital stay, previous treatment such as surgical procedures, immunosuppressive agents, chemotherapeutic agents, radiation therapy, parenteral nutrition, mechanical ventilation, renal replacement therapy, blood transfusion), and outcomes (length of hospital stay, length of ICU stay, occurrence of septic shock and 28-day mortality). The microbiological data were also collected including likely source of BSI (identified by treating doctors), monomicroorganism/poly-microorganisms, and sensitivity to antibiotics. If the source of a BSI could not be attributed to any known source, it was classified as a primary BSI [12].

\section{Species identification and antibiotic sensitivity test}

Blood was cultured using a BacT/ALERT 3D system (Becton-Dickinson, Sparks, MD, USA) in the microbiology laboratory. Species identification was performed using Bruker Daltonics DataAnalysis. Antibiotic susceptibility testing was performed using the VITEK 2 (Card number: AST-GN16; AST-GP67) system or the Kirby- 


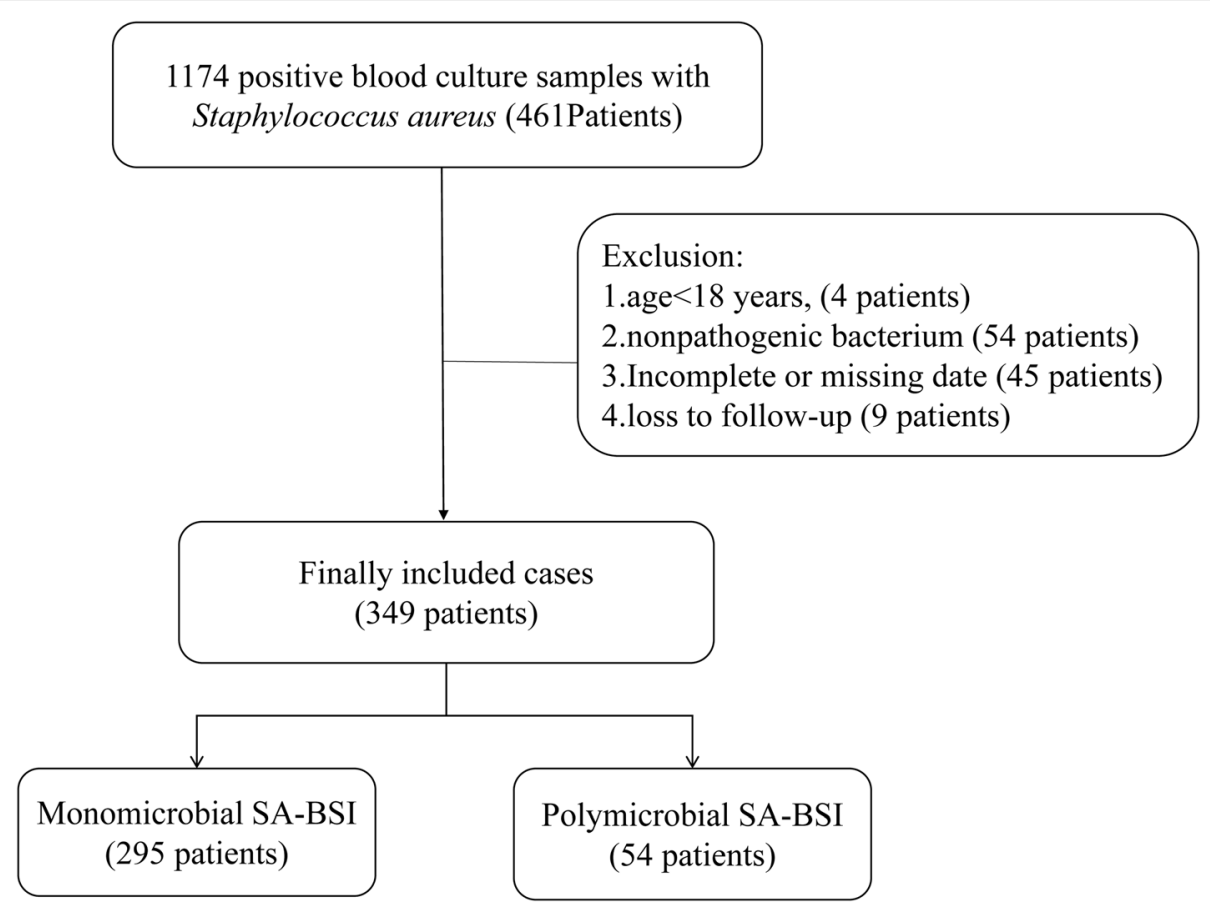

Fig. 1 Flowchart of study participant enrollment. Abbreviations: SA-BSI, Staphylococcus aureus bloodstream infections

Bauer Disk Diffusion method (Oxoid, UK) according to the recommendations proposed by the Clinical and Laboratory Standards Institute (CLSI).

\section{Definitions}

Diagnosis of SA-BSI was based on CDC definitions for Bloodstream Infection Events [12]. Onset of BSI was defined as the time when the blood culture was collected. Polymicrobial SA-BSI was defined as the simultaneous isolations of $S$. aureus and one or more other organisms from blood cultures [10]. Nosocomial BSI was defined as the first positive blood culture obtained $\geq 48 \mathrm{~h}$ after hospital admission and with no evidence of infection at admission [13, 14]. Infective endocarditis was defined using the modified Duke criteria [15]. Appropriate antimicrobial therapy was considered early when administered within $24 \mathrm{~h}$ after the first blood culture that yielded S. aureus had been obtained [16], whereas therapy was considered delayed when more than $24 \mathrm{~h}$ had elapsed [16]. Neutropenia was defined as absolute neutrophil counts of $1000 / \mathrm{mm}^{3}$ or below when bacteremia occurred. Sepsis and septic shock were defined according to the new definition of Sepsis-3 [17]. Secondary BSI was defined as a BSI that is thought to be seeded from a site-specific infection at another body site [12].

\section{Statistical analysis}

Statistical analysis was performed with SPSS 20.0 software (IBM Corp, Armonk, NY, USA). Continuous variables were presented as mean \pm standard deviation if normally distributed, and as median and interquartile range (IQR) if non-normally distributed. Continuous variables were compared by Student $t$ test or MannWhitney $U$ test and enumeration variables were compared by Pearson $x^{2}$ or Fisher exact test, where appropriate. Variables that had significance at a $p<0.05$ level in the univariate analysis were considered candidates for the building of stepwise logistic regression multivariable models. A two-tailed $p<0.05$ was considered statistically significant.

\section{Results}

Demographic and clinical characteristics

The demographic and clinical characteristics of these patients were summarized in Table 1 . The median age was 59 years (IQR, 45.5-68), and 69.6\% (243/349) were male. Trauma was the most common comorbidity (20.9\%), followed by diabetes mellitus (20.1\%). There were no significant differences in age or gender between the two groups. In terms of co-morbidities, a significantly high percentage of trauma, burn injuries, or cerebrovascular accident was observed in patients with polymicrobial SA-BSI (all $p<0.05$ ). In comparison with monomicrobial SA-BSI, patients with polymicrobial SA-BSI presented a more severe condition, evidenced by a higher APACHE II score (median, 15 vs. $12, p<0.01$ ), a higher SOFA score (median, 5 vs. $3, p=0.01$ ) and a higher Pitt Bacteremia Score (median, 3.5 vs $1, p<0.01$ ), and 
Table 1 Baseline characteristics of patients with polymicrobial and monomicrobial Staphylococcus aureus bloodstream infection

\begin{tabular}{|c|c|c|c|c|}
\hline Characteristics & $\begin{array}{l}\text { Total } \\
(n=349)\end{array}$ & $\begin{array}{l}\text { Mono-SA-BSI } \\
(n=295)\end{array}$ & $\begin{array}{l}\text { Poly-SA-BSI } \\
(n=54)\end{array}$ & $p$-value \\
\hline Age, median years (IQR) & $59.00(45.50,68.00)$ & $60.00(48,68.00)$ & $51.00(40.50,69.00)$ & 0.06 \\
\hline Male sex & $243(69.6 \%)$ & $202(68.5 \%)$ & $41(75.9 \%)$ & 0.27 \\
\hline \multicolumn{5}{|l|}{ Co-morbidities } \\
\hline Diabetes mellitus & $70(20.1 \%)$ & $61(20.7 \%)$ & $9(16.7 \%)$ & 0.50 \\
\hline Chronic kidney disease & $31(8.9 \%)$ & $27(9.2 \%)$ & $4(7.4 \%)$ & 0.88 \\
\hline Chronic liver disease & $16(4.6 \%)$ & $14(4.7 \%)$ & $2(3.7 \%)$ & 1 \\
\hline COPD or Severe asthma & $7(2 \%)$ & $7(2.4 \%)$ & $0(0 \%)$ & 0.60 \\
\hline Chronic cardiac insufficiency & $25(7.2 \%)$ & $22(7.5 \%)$ & $3(5.6 \%)$ & 0.83 \\
\hline Solid tumor & $57(16.3 \%)$ & $48(16.3 \%)$ & $9(16.7 \%)$ & 0.94 \\
\hline Trauma & $73(20.9 \%)$ & $54(18.3 \%)$ & $19(35.2 \%)$ & 0.01 \\
\hline Burn injury & $45(12.9 \%)$ & $30(10.2 \%)$ & $15(27.8 \%)$ & 0.00 \\
\hline Cerebrovascular accident & $39(11.2 \%)$ & $28(9.5 \%)$ & $11(20.4 \%)$ & 0.02 \\
\hline $\mathrm{CCl}$, median (IQR) & $3(1,5)$ & $3(2,5)$ & $2(1,4.25)$ & 0.08 \\
\hline APACHE II score, median (IQR) & $12(9,17)$ & $12(8,16)$ & $15(11,22)$ & 0.00 \\
\hline SOFA score, median (IQR) & $4(2,6)$ & $3(2,5)$ & $5(2.75,10)$ & 0.01 \\
\hline Pitt Bacteremia Score, median (IQR) & $2(1,4)$ & $1(1,3)$ & $3.5(2,6)$ & 0.00 \\
\hline \multicolumn{5}{|l|}{ Hospitalization ward } \\
\hline ICU stay & $131(37.5 \%)$ & $93(31.5 \%)$ & $38(70.4 \%)$ & 0.00 \\
\hline \multicolumn{5}{|l|}{ Previous treatment } \\
\hline Parenteral nutrition & $122(35 \%)$ & 91 (30.8\%) & $31(57.4 \%)$ & 0.00 \\
\hline Mechanical ventilation & $112(32.1 \%)$ & $76(25.8 \%)$ & $36(66.7 \%)$ & 0.00 \\
\hline Antibiotic exposure & $302(86.5 \%)$ & $251(85.1 \%)$ & $51(94.4 \%)$ & 0.06 \\
\hline Surgery & $124(35.5 \%)$ & $92(31.2 \%)$ & $32(59.3 \%)$ & 0.00 \\
\hline Chemotherapy/radiation & $13(3.7 \%)$ & $13(4.4 \%)$ & $0(0 \%)$ & 0.23 \\
\hline Renal replacement therapy & $43(12.3 \%)$ & $37(12.5 \%)$ & $6(11.1 \%)$ & 0.77 \\
\hline Blood transfusion & $58(16.6 \%)$ & $37(12.5 \%)$ & $21(38.9 \%)$ & 0.00 \\
\hline \multicolumn{5}{|l|}{ Invasive devices } \\
\hline central line catheter & $167(47.9 \%)$ & $132(44.7 \%)$ & $35(64.8 \%)$ & 0.01 \\
\hline Indwelling urinary catheter & $175(50.1 \%)$ & $132(44.7 \%)$ & $43(79.6 \%)$ & 0.00 \\
\hline Intraperitoneal drainage & $33(9.5 \%)$ & $26(8.8 \%)$ & $7(13 \%)$ & 0.34 \\
\hline Prior hospital stay, median days (IQR) & $8(2,20)$ & $7(1,19)$ & $13(7,32.5)$ & 0.00 \\
\hline Nosocomial infection & $266(76.2 \%)$ & $214(72.5 \%)$ & $52(96.3 \%)$ & 0.00 \\
\hline Neutropenia & 7 (2.0\%) & $6(2.0 \%)$ & 1 (1.9\%) & 1 \\
\hline
\end{tabular}

Abbreviations: IQR Interquartile range, COPD Chronic obstructive pulmonary disorder, CCI Charlson Comorbidity Index, SOFA Sequential organ failure assessment, APACHE Acute physiology and chronic health evaluation, ICU Intensive care unit, SA-BSI Staphylococcus aureus bloodstream infection

displayed more need of ICU admission (70.4\% vs. $31.5 \%$, $p<0.01)$ or invasive mechanical ventilation $(66.7 \%$ vs. $25.8 \%, p<0.01)$. Compared with monomicrobial SA-BSI, patients with polymicrobial SA-BSI had a greater proportion of receiving parenteral nutrition $(57.4 \%$ vs. $30.8 \%, p<0.01)$, more need of blood transfusion $(38.9 \%$ vs. $12.5 \%, p<0.01$ ), significant increases in central line indwelling and urinary catheter indwelling (64.8\% vs. $44.7 \%, p<0.01$; and $79.6 \%$ vs. $44.7 \%, p<0.01$ ), more surgery $(59.3 \%$ vs $31.2 \%, p<0.01)$, and more nosocomial infections (96.3\% vs. $72.5 \%, p<0.01)$. In addition, a longer hospital stay before BSI onset was observed in patients with polymicrobial SA-BSI than monomicrobial SA-BSI (median days, 13 vs. $7, p<0.01$ ).

\section{Biological indicators}

A comparison of biological indicators between polymicrobial SA-BSI and monomicrobial SA-BSI was shown in Table 2. In comparison with monomicrobial SA-BSI, patients with polymicrobial SA-BSI had a lower 
Table 2 Comparison of biological indicators between groups of monomicrobial SA-BSI and polymicrobial SA-BSI

\begin{tabular}{|c|c|c|c|c|}
\hline Biological indicators & $\begin{array}{l}\text { Total } \\
(n=349)\end{array}$ & $\begin{array}{l}\text { Mono-SA-BSI } \\
(n=295)\end{array}$ & $\begin{array}{l}\text { Poly-SA-BSI } \\
(n=54)\end{array}$ & $p$-value \\
\hline Temperature $\left({ }^{\circ} \mathrm{C}\right)(\mathrm{IQR})$ & $39.0(38.6,39.5)$ & $39(38.6,39.5)$ & $39(38.575,39.5)$ & 0.51 \\
\hline \multicolumn{5}{|l|}{ Blood routine test } \\
\hline $\mathrm{WBC}\left(\times 10^{9} / \mathrm{L}\right)(\mathrm{IQR})$ & $10.1(6.8,14.35)$ & $10.3(6.9,14.2)$ & $9.75(5.5,16.65)$ & 0.94 \\
\hline Hematocrit (\%) (IQR) & $28.9(24.05,33.5)$ & $29.2(24.6,33.9)$ & $26.95(22.6,31.2)$ & 0.01 \\
\hline Platelet $\left(\times 10^{9} / \mathrm{L}\right)(\mathrm{IQR})$ & $171(102.5247 .5)$ & $175(101,248)$ & $145.5(103,248)$ & 0.37 \\
\hline ANC (IQR) & $8.15(5.56,12.74)$ & $8.1(5.6,12.35)$ & $10.545(5.44,15.79)$ & 0.13 \\
\hline \multicolumn{5}{|l|}{ Liver and kidney function } \\
\hline Albumin $(g / L)$ (mean \pm S.D.) & $29.56 \pm 5.66$ & $30.216 \pm 5.48$ & $29.44 \pm 5.69$ & 0.36 \\
\hline GPT (U/L) (IQR) & $31(17,57)$ & $30(17,53)$ & $41(24.5,73)$ & 0.01 \\
\hline GOT (U/L) (IQR) & $30(21,50)$ & $28(20,47)$ & $36(27.5,60.25)$ & 0.00 \\
\hline $\mathrm{ALP}(\mathrm{U} / \mathrm{L})(\mathrm{IQR})$ & $101(74,151)$ & $103(74,150)$ & $99.5(76.75,152.25)$ & 0.97 \\
\hline$\gamma-G T(U / L)(I Q R)$ & $45(26.0,95)$ & $44(25.0,96.0)$ & $56.0(27.25,89)$ & 0.42 \\
\hline LDH (U/L) (IQR) & $254(197,342)$ & $248(195,321)$ & $343(227.5405)$ & 0.00 \\
\hline TBil $(\mu \mathrm{mol} / \mathrm{L})(\mathrm{QQR})$ & $12.8(9,20.35)$ & $12.7(9,19.6)$ & $14.85(9.38,26.43)$ & 0.26 \\
\hline $\mathrm{SCr}(\mu \mathrm{mol} / \mathrm{L})(\mathrm{IQR})$ & $59(44.5,95.5)$ & $59(45,96)$ & $65.5(42.25,96.25)$ & 0.94 \\
\hline $\mathrm{PCT}(\mathrm{ng} / \mathrm{ml})(\mathrm{IQR})$ & $0.46(0.18,1.31)$ & $0.46(0.18,1.29)$ & $0.53(0.22,2.08)$ & 0.36 \\
\hline
\end{tabular}

Abbreviations: SA-BSI Staphylococcus aureus bloodstream infections, IQR Interquartile range, WBC White blood count, ANC Absolute neutrophil count, GPT Glutamicpyruvic transaminase, GOT Glutamic-oxaloacetic transaminase, ALP Alkaline phosphatase, $\gamma-G T$ Gamma glutamyl transpeptidase, $\underline{L D H}$ Lactic dehydrogenase, TBil Total bilirubin, SCr Serum creatinine, PCT Procalcitonin

Table 3 Multivariable logistic regression of factors associated with polymicrobial Staphylococcus aureus bloodstream infections

\begin{tabular}{|c|c|c|c|c|}
\hline Variable & $\begin{array}{l}\text { Unadjusted } \\
\text { OR(95\%Cl) }\end{array}$ & $p$-value & $\begin{array}{l}\text { Adjusted } \\
\text { OR(95\%Cl) }\end{array}$ & $p$-value \\
\hline \multicolumn{5}{|l|}{ Co-morbidities } \\
\hline Trauma & $2.42(1.29,4.56)$ & 0.01 & & \\
\hline Burn injury & $3.40(1.68,6.88)$ & 0.00 & $7.04(1.71,28.94)$ & 0.01 \\
\hline Cerebrovascular accident & $2.44(1.13,5.26)$ & 0.02 & & \\
\hline APACHE II score & $1.08(1.04,1.12)$ & 0.00 & & \\
\hline SOFA score & $1.11(1.04,1.18)$ & 0.00 & & \\
\hline Pitt Bacteremia Score & $1.29(1.15,1.44)$ & 0.00 & & \\
\hline ICU stay & $5.16(2.74,9.72)$ & 0.00 & & \\
\hline \multicolumn{5}{|l|}{ Previous treatment } \\
\hline Parenteral nutrition & $3.02(1.67,5.47)$ & 0.00 & & \\
\hline Previous surgery & $3.21(1.77,5.83)$ & 0.00 & & \\
\hline Prior Blood transfusion & $4.44(2.32,8.47)$ & 0.00 & $2.72(1.14,6.50)$ & 0.03 \\
\hline Central venous catheter & $2.28(1.24,4.16)$ & 0.01 & & \\
\hline Mechanical ventilation & $5.76(3.09,10.75)$ & 0.00 & $3.11(1.16,8.30)$ & 0.02 \\
\hline Indwelling urinary catheter & $4.83(2.40,9.73)$ & 0.00 & & \\
\hline Nosocomial infection & $9.84(2.34,41.34)$ & 0.00 & & \\
\hline Prior hospital stay & $1.02(1.01,1.03)$ & 0.01 & $1.02(1.00,1.03)$ & 0.02 \\
\hline \multicolumn{5}{|l|}{ Source of BSIs } \\
\hline Pneumonia & $2.62(1.44,4.78)$ & 0.00 & $4.22(1.69,10.51)$ & 0.00 \\
\hline Central venous catheter & $0.31(0.11,0.88)$ & 0.03 & & \\
\hline MRSA & $2.36(1.14,4.88)$ & 0.02 & & \\
\hline
\end{tabular}


hematocrit (median \%, 26.95 vs. 29.2, $p<0.01$ ), a worse liver function evidenced by significant increases in Glutamic-pyruvic transaminase (GPT) (median U/L, 41 vs. 30, $p=0.01$ ), Glutamic-oxaloacetic transaminase (GOT) (median U/L, 36 vs. 28, $p<0.01$ ) and Lactic dehydrogenase (LDH) (median U/L, 343 vs. 248, $p<0.01$ ). However, there was no significant difference in procalcitonin between the two groups.

\section{Independent risk factors for polymicrobial SA-BSI}

As shown in Table 3, multivariate logistic regression model analysis showed that the independent risk factors of polymicrobial SA-BSI were burn injury (adjusted odds ratio [aOR], 7.04; 95\% confidence interval [CI], 1.7128.94), prior blood transfusion (aOR, 2.72; 95\% CI, 1.146.50), mechanical ventilation (aOR, 3.11; 95\% CI, 1.168.30), pneumonia as a primary site of infection (aOR, 4.22; 95\% CI, 1.69-10.51), and the days of prior hospital stay before onset of BSI (aOR, 1.02; 95\% CI, 1.00-1.03).

\section{Bacteriology and sources of polymicrobial SA-BSI}

The isolated pathogens were shown in Fig. 2. A total of 61 microorganisms other than $S$. aureus were isolated from 54 polymicrobial SA-BSI cases, with two microorganisms accounting for $87 \%$ (47/54) and three microorganisms for $13 \%$ (7/54). The most common copathogen was Gram-negative bacteria (54.1\%), followed by Gram-positive bacteria (36.1\%) and fungi (9.8\%). In terms of a specific microorganism, the most frequent pathogen was Acinetobacter baumannii (A. baumannii) (27.9\%), followed by Enterococcus spp. (26.2\%). Candida spp. was observed in only $11.1 \%(6 / 54)$ of patients, representing $9.8 \%$ of all isolates (Fig. 2).

The source of SA-BSI was mainly from pneumonia $(26.6 \%, 93 / 349)$, followed by skin/soft tissue infection (24.6\%, 86/349), and central venous catheter $(18.6 \%, 65 /$ 349) (Table 4). Compared with monomicrobial SA-BSI, polymicrobial SA-BSI had more source from pneumonia (44.4\% vs. $23.4 \%, p<0.01)$, in which the polymicrobial SA-BSI caused by hospital-acquired pneumonia was significantly higher than that caused by communityacquired pneumonia $(91.7 \%$ vs. $8.3 \%, p=0.02)$, whereas monomicrobial SA-BSI had more source from central venous catheter $(20.7 \%$ vs. $7.4 \%, p=0.04)$.

\section{Antibiotic resistance and appropriate therapy}

In comparison with monomicrobial SA-BSI, the ratio of resistance of Staphylococcus aureus to cefoxitin, ciprofloxacin, levofloxacin, moxifloxacin, oxacillin, and tetracycline were significantly higher in polymicrobial SA-BSI

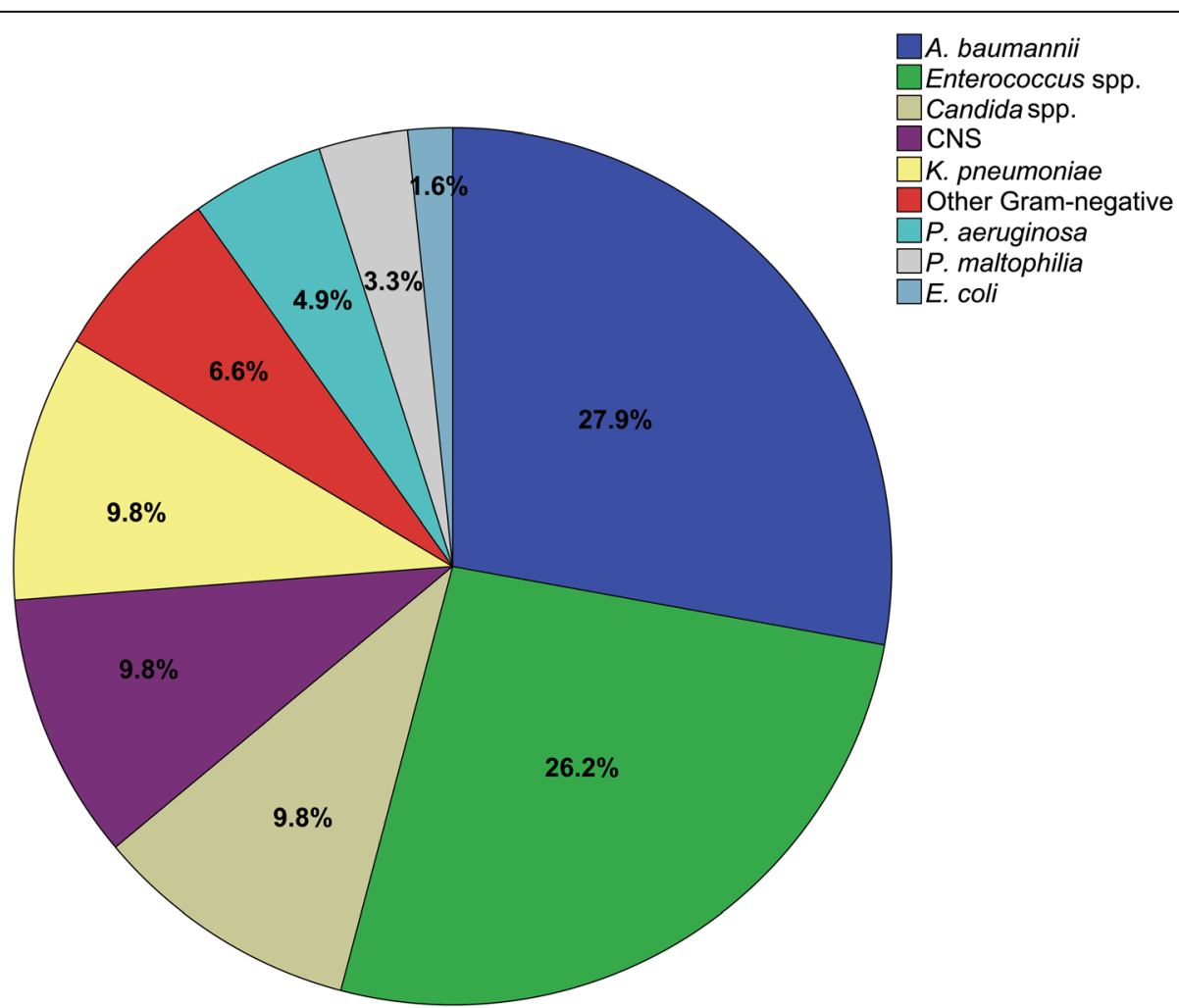

Fig. 2 Distribution of the additional organisms in polymicrobial Staphylococcus aureus bloodstream infections. Abbreviations: E. coli, Escherichia coli; A. baumannii, Acinetobacter baumannii; CNS, Coagulase-negative Staphylococcus; K. pneumoniae, Klebsiella pneumoniae; P. aeruginosa, Pseudomonas aeruginosa; P. maltophilia, Pseudomonas maltophilia 
Table 4 Comparison of the microbiological characteristics with monomicrobial SA-BSI and polymicrobial SA-BSI

\begin{tabular}{|c|c|c|c|c|}
\hline & $\begin{array}{l}\text { Total } \\
(n=349)\end{array}$ & $\begin{array}{l}\text { Mono-SA-BSI } \\
(n=295)\end{array}$ & $\begin{array}{l}\text { Poly-SA-BSI } \\
(n=54)\end{array}$ & $p$-value \\
\hline \multicolumn{5}{|l|}{ Source of BSIs } \\
\hline Pneumonia & $93(26.6 \%)$ & $69(23.4 \%)$ & $24(44.4 \%)$ & 0.00 \\
\hline Hospital-acquired pneumonia & $69(74.2 \%)$ & $47(68.1 \%)$ & $22(91.7 \%)$ & 0.02 \\
\hline Community-acquired pneumonia & $24(25.8 \%)$ & $22(31.9 \%)$ & $2(8.3 \%)$ & \\
\hline Skin and Soft tissue infection & $86(24.6 \%)$ & $69(23.4 \%)$ & $16(29.6 \%)$ & 0.33 \\
\hline Central venous catheter & $65(18.6 \%)$ & $61(20.7 \%)$ & $4(7.4 \%)$ & 0.04 \\
\hline Intra-abdominal & $42(12 \%)$ & $34(11.5 \%)$ & $8(14.8 \%)$ & 0.50 \\
\hline Primary BSI & $28(7.7 \%)$ & $27(9.2 \%)$ & $1(1.9 \%)$ & 0.10 \\
\hline Bone and joint & $14(4.0 \%)$ & $14(4.7 \%)$ & $0(0 \%)$ & 0.14 \\
\hline Endocarditis & $11(3.2 \%)$ & $11(3.7 \%)$ & $0(0 \%)$ & 0.23 \\
\hline Urinary tract infection & $9(2.6 \%)$ & $8(2.7 \%)$ & $1(1.9 \%)$ & 0.58 \\
\hline Others* & $2(0.6 \%)$ & $2(0.7 \%)$ & $0(0 \%)$ & 0.71 \\
\hline MRSA & $236(67.6 \%)$ & $192(65.1 \%)$ & $44(81.5 \%)$ & 0.02 \\
\hline Delayed antibiotic therapy & $29(8.3 \%)$ & $24(8.1 \%)$ & $5(9.3 \%)$ & 0.78 \\
\hline \multicolumn{5}{|l|}{ Antibiotic resistance ${ }^{a}$} \\
\hline Cefoxitin (115 vs 25$)^{\mathrm{b}}$ & $114(81.4 \%)$ & $89(77.4 \%)$ & $25(100 \%)$ & 0.01 \\
\hline Ciprofloxacin (237 vs 42$)^{\mathrm{b}}$ & $132(47.3 \%)$ & $96(40.5 \%)$ & $36(85.5 \%)$ & 0.00 \\
\hline Clindamycin (295 vs 54) ${ }^{\mathrm{b}}$ & $127(36.4 \%)$ & $110(37.3 \%)$ & $17(31.5 \%)$ & 0.42 \\
\hline Erythromycin (295 vs 54) ${ }^{\mathrm{b}}$ & $203(58.2 \%)$ & $167(56.6 \%)$ & $36(66.7 \%)$ & 0.17 \\
\hline Gentamicin (295 vs 54$)^{\mathrm{b}}$ & $58(16.6 \%)$ & $50(16.9 \%)$ & $8(14.8 \%)$ & 0.71 \\
\hline Levofloxacin (261 vs 49) ${ }^{b}$ & $161(51.9 \%)$ & $118(45.2 \%)$ & $43(87.8 \%)$ & 0.00 \\
\hline Linezolid (293 vs 54) & $46(13.3 \%)$ & $37(12.6 \%)$ & $9(16.7 \%)$ & 0.42 \\
\hline Moxifloxacin (287 vs 51) ${ }^{\mathrm{b}}$ & $158(46.7 \%)$ & $117(40.8 \%)$ & 41 (80.4\%) & 0.00 \\
\hline Nitrofurantoin (255 vs 50$)^{b}$ & $1(0.3 \%)$ & $1(0.4 \%)$ & $0(0 \%)$ & 1 \\
\hline Oxacillin (295 vs 54) ${ }^{\mathrm{b}}$ & $236(67.6 \%)$ & $192(65.1 \%)$ & $44(81.5 \%)$ & 0.02 \\
\hline Penicillin (295 vs 54$)^{b}$ & $333(95.4 \%)$ & $279(94.6 \%)$ & $54(100 \%)$ & 0.08 \\
\hline Quinupristin/Dalfopristin (295 vs 54) b $^{\mathrm{b}}$ & $44(12.6 \%)$ & $36(12.2 \%)$ & $8(14.8 \%)$ & 0.60 \\
\hline Rifampin (242 vs 43$)^{b}$ & $9(3.2 \%)$ & $8(3.3 \%)$ & $1(2.3 \%)$ & 1 \\
\hline Tetracycline $(230 \text { vs } 50)^{b}$ & $148(52.9 \%)$ & $112(48.7 \%)$ & $36(72 \%)$ & 0.00 \\
\hline Vancomycin (278 vs 49) ${ }^{\mathrm{b}}$ & $48(14.3 \%)$ & $39(13.6 \%)$ & 9 (18.4\%) & 0.38 \\
\hline
\end{tabular}

*oromaxillo-facial region and prosthetic device

${ }^{a}$ Not all agents listed tested in all isolates

${ }^{\mathrm{b}}$ The numbers in parentheses represent the total numbers of Staphylococcus aureus isolates performed susceptibility test

Abbreviations: BSIs Bloodstream infections, MRSA Methicillin-resistant Staphylococcus aureus, SA-BSI Staphylococcus aureus bloodstream infections

Table 5 Comparison of outcome between monomicrobial SA-BSI and polymicrobial SA-BSI

\begin{tabular}{|c|c|c|c|c|}
\hline Prognostic indicators & $\begin{array}{l}\text { Total } \\
(n=349) \\
\end{array}$ & $\begin{array}{l}\text { Mono-SA-BSI } \\
(n=295)\end{array}$ & $\begin{array}{l}\text { Poly-SA-BSI } \\
(n=54)\end{array}$ & $p$-value \\
\hline Total Hospitalization days (M) (IQR) & $30(17,54.5)$ & $28(15,49)$ & $50(21.25,85.75)$ & 0.00 \\
\hline Total ICU residence days (M) (IQR) & $1(0,18)$ & $0(0,12)$ & $23(6.25,49.25)$ & 0.00 \\
\hline Cause sepsis & $288(82.5 \%)$ & $249(84.4 \%)$ & $39(72.2 \%)$ & 0.03 \\
\hline Cause Septic shock $(n, \%)$ & $35(10 \%)$ & $26(8.8 \%)$ & $9(16.7 \%)$ & 0.08 \\
\hline 7 day total mortality rate $(n, \%)$ & $31(8.9 \%)$ & $22(7.5 \%)$ & $9(16.7 \%)$ & 0.03 \\
\hline 14 day total mortality rate $(n, \%)$ & $46(14.2 \%)$ & $33(11.2 \%)$ & $13(24.1 \%)$ & 0.01 \\
\hline 28 day total mortality rate $(n, \%)$ & $61(17.5 \%)$ & $45(15.3 \%)$ & $16(29.6 \%)$ & 0.01 \\
\hline In-hospital mortality (n,\%) & $71(20.3 \%)$ & $54(18.3 \%)$ & y (31.5\%) & 0.03 \\
\hline
\end{tabular}


groups (Table 4). Of note, methicillin-resistant Staphylococcus aureus (MRSA) was significantly more frequent in patients with polymicrobial than monomicrobial SABSI $(81.5 \%$ vs. $65.1 \%, p=0.02)$. In addition, a total of 8.3\% (29/349) patients did not receive appropriate therapy within $24 \mathrm{~h}$ after the release of antibiotic susceptibility results, but there was no difference between the two groups $(8.1 \%$ vs. $9.3 \%, p=0.78)$ (Table 4$)$.

\section{Outcomes}

In comparison with monomicrobial SA-BSI, patients with polymicrobial SA-BSI had a longer length of hospital stay [median days, $28(15-49)$ vs. 50(21.25-85.75), $p<0.01$ ], and ICU stay [median days, $0(0-12)$ vs. 23(6.25-49.25), $p<0.01$ ] (Table 5). Sepsis occurred in $72.2 \%$ of polymicrobial SA-BSI and in $84.4 \%$ of monomicrobial SA-BSI $(p=0.03)$, whereas the occurrence rate of septic shock in patients with polymicrobial SA-BSI was almost two-fold higher than that with monomicrobial SA-BSI ( $16.7 \%$ vs. $8.8 \%, p=0.08)$. The overall in-hospital crude mortality rate was $20.3 \%$, which was significantly higher in patients with polymicrobial SA-BSI than that in patients with monomicrobial SA-BSI (31.5\% vs. $18.3 \%$, $p=0.03)$. Like the 28 -day mortality (29.6\% vs. $15.3 \%, p=$ 0.01 ), the 7-day and 14-day mortalities in patients with polymicrobial SA-BSI were also significantly higher than those with monomicrobial SA-BSI $(16.7 \%$ vs. $7.5 \%, p=$ $0.03 ; 24.1 \%$ vs. $11.2 \%, p=0.01$, respectively) (Table 5 ), which were consistent with the results from the survival curves of patients in both groups (Fig. 3).

\section{Discussion}

In the current study, several important results were found. First, polymicrobial SA-BSI are not rare among Staphylococcus aureus bacteremia. Second, some risk factors were found to be associated with polymicrobial SA-BSI as shown in Table 1. Moreover, burn injury, prior blood transfusion, mechanical ventilation, pneumonia as a primary site of infection, and length of prior hospital stay were independent risk factors for polymicrobial SA-BSI (Table 3). Third, A. baumannii was the most common co-pathogen in polymicrobial SA-BSI, followed by Enterococcus spp.. Last, patients with polymicrobial SA-BSI might have worse outcomes including higher occurrence of septic shock, prolonged ICU stay,

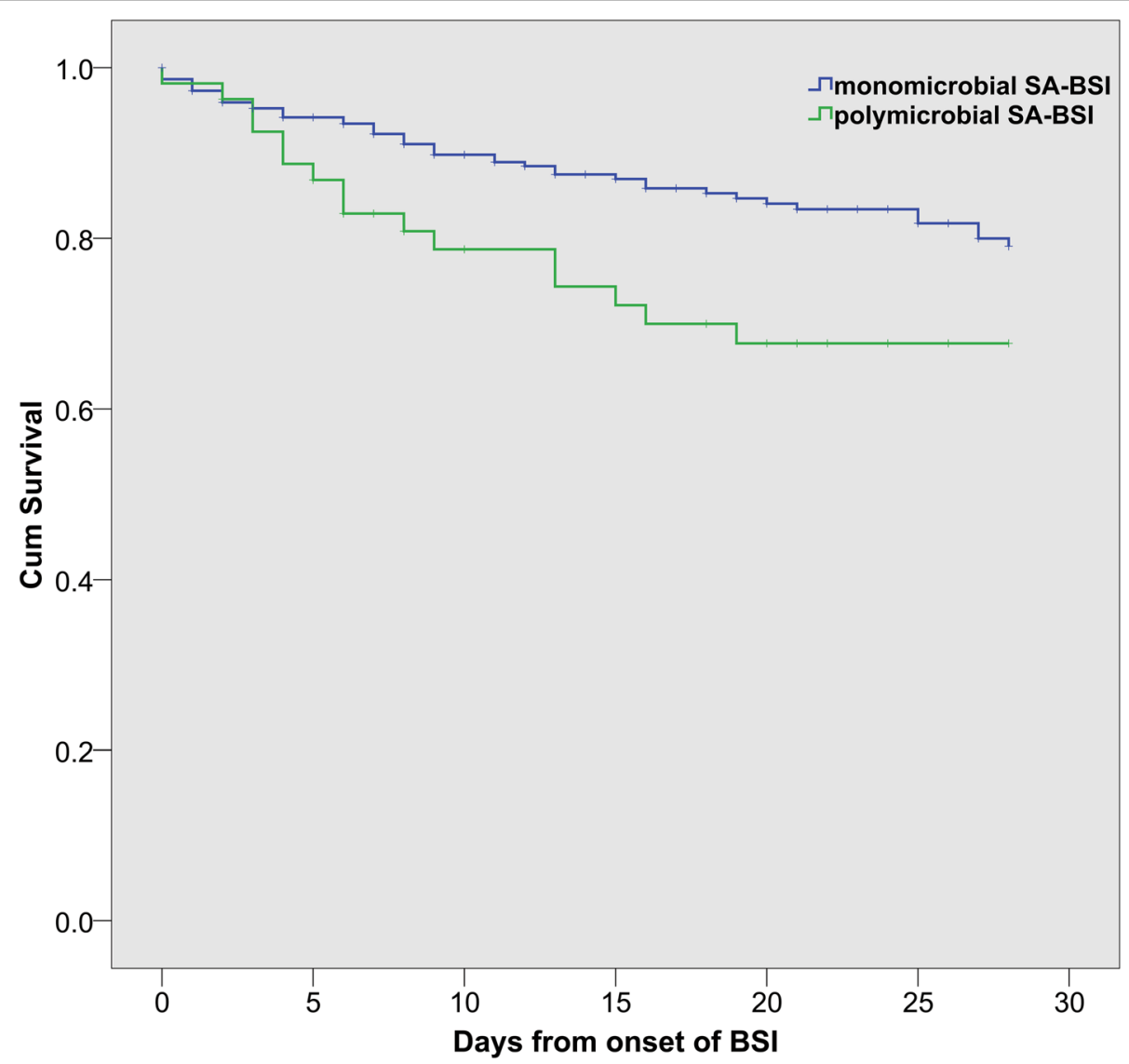

Fig. 3 Kaplan-Meier estimates of survival in patients with polymicrobial Staphylococcus aureus bloodstream infections and monomicrobial Staphylococcus aureus bloodstream infections. Abbreviations: SA-BSI, Staphylococcus aureus bloodstream infections 
and hospital stay as well as higher mortality in comparison with monomicrobial SA-BSI.

A high proportion of $15.5 \%$ was polymicrobial SA-BSI among SA-BSI in the current study, which was consistent with previous studies that polymicrobial bacteremia accounts for 5-20\% of bloodstream infection [7, 18-20]. In Park's report [10], the polymicrobial SA-BSI was accounted for $9.6 \%(44 / 456)$ of all episodes of BSI in a tertiary referral center of Korea. A 6.1\% (93/1537) frequency of polymicrobial SA-BSI was reported in a 772bed teaching hospital in Michigan in Khatib's study [11]. In our recently previous study [21], we also found that $34.8 \%$ cases $(157 / 451)$ with enterococcal bloodstream infections were mixed with other pathogens like CNS, $A$. baumannii and Klebsiella pneumoniae. These studies suggest that the proportion of polymicrobial bloodstream infections is not rare, which deserves the attention of clinicians.

We found that clinical and demographic were different between the monomicrobial and polymicrobial groups. This suggests that many factors were associated with polymicrobial SA-BSI (Table 1). One of them appears to be patients with traumatic or burn injuries leading to neurologic deficits, prolonged ventilation and ICU stays, and frequent antibiotics (as evidenced by higher resistance rates, Table 4). Many independent risk factors for polymicrobial SA-BSI were observed in our current study including burn injury, prior blood transfusion, mechanical ventilation, pneumonia as a primary site of infection, and length of prior hospital stay. A previous study has shown that more than $12 \%$ of burn patients suffered from polymicrobial BSI [22]. Our previous study focusing on enterococcal bloodstream infection also confirmed that burn was an independent risk factor for mixed-enterococcal bloodstream infections [21]. As described in previous studies [23-25], burn patients were at a high risk of BSI as they encountered alterations in cellular and humoral immune responses, extensive skin barrier disruption, high possibility of gastrointestinal bacterial translocation, prolonged hospitalization, and invasive diagnostic/therapeutic procedures. Therefore, as the most common colonizing pathogens of the skin, $S$. aureus is more likely to invade the blood through the skin of burn patients and caused bloodstream infections together with other pathogens.

Blood transfusion was an independent risk factor of polymicrobial SA-BSI in our research, which was consistent with previous study, showing that transfusion of red blood cells and platelets was associated with the onset of secondary bacterial infection in sepsis patients [26]. This is partly explained that transfusion can cause persistent immune dysfunction in mouse model of sepsis [27] and the fact that immunosuppressive patients are more likely to have polymicrobial BSI $[20,28]$. In addition, patients with polymicrobial SA-BSI were more severe evidenced by high APACHE II score and SOFA score (Table 1), which suggested that these patients were more like in the immunosuppressive state.

Our study also showed mechanical ventilation and pneumonia as a primary site of infection were independent factors of polymicrobial SA-BSI. As shown in the current study, pneumonia was the most common source of SA-BSI and was significantly more frequent among patients with polymicrobial than monomicrobial SA-BSI, which was consistent with Sancho's study showing that lower respiratory tract was the main source of BSI [9]. In contrast, a previous study [10] has shown that intraabdominal infections were the most common source of polymicrobial infection, but a selected bias might be existed as a high proportion (7\%) of biliary tract disease was observed in their institution. Compared with monomicrobial SA-BSI, our study showed that polymicrobial SA-BSI had a higher APACHE II score, a higher SOFA score, a higher Pitt Bacteremia score, and more frequent admission in ICU (Table 1). These results suggested that polymicrobial SA-BSI was associated with more severity conditions. Critical patients with pneumonia might tend to develop respiratory failure and require mechanical ventilation [8], and mechanical ventilation can increase the incidence of ventilators-associated complications, such as ventilators associated pneumonia (VAP) [29, 30], leading to recurrent lung infections and the increased possibility of polymicrobial bloodstream infections. Like in our and Sancho's study $[9,21]$, an independent association between the days of prior hospital stay and polymicrobial infection was also observed in the current study. It can be inferred that such patients would be more predisposed to health care exposure and risks for contracting polymicrobial infection. Thus, it might be important to reduce unnecessary interventions and shorten the length of stay for patients.

The most common co-pathogen in polymicrobial SABSI was A. baumannii (27.9\%) in the current study. In fact, the high proportion of A. baumannii as copathogens in polymicrobial SA-BSI is also indirectly reflected by the evidence that pneumonia as a primary site of infection and mechanical ventilation were independent risk factors for polymicrobial SA-BSI in our current study, as A. baumannii was frequently associated with pneumonia infection especially for VAP [31, 32]. By the way, A. baumannii as the most common pathogen in post-neurosurgical intracranial infections accounted for 38.8 percentage in our previous study [33]. These results mean A. baumannii is a real threat for hospitalacquired infection. Patients with polymicrobial SA-BSI might have worse outcomes than those with monomicrobial SA-BSI, including prolonged lengths of ICU stay and hospital stay, the 28-day mortality, which were 
consistent with previous reports $[9,10]$. Although early appropriate antimicrobial therapy has been shown to reduce mortality among bacteremia patients [34], there was no difference in delayed antibiotic therapy between the two groups in our study. The worse outcomes of polymicrobial SA-BSI in our study were possibly associated with the following factors: (1) the proportion of septic shock in patients with polymicrobial SA-BSI was two-fold higher than that with monomicrobial SA-BSI $(16.7 \%$ vs. $8.8 \%)$, though there was no statistical difference. (2) Interestingly, MRSA was significantly more frequent in patients with polymicrobial than monomicrobial SA-BSI $(81.5 \%$ vs. $65.1 \%, p=0.018)$. As shown in a previous meta-analysis, methicillin resistance is associated with increased mortality in patients with $S$. aureus bacteremia [35]. (3) A high proportion of secondary bloodstream infections was observed in polymicrobial SA-BSI than that in monomicrobial SA-BSI $(90.7 \%$ vs. 70.2\%). Previous study has shown that the risk of mortality associated with primary bacteremia like the catheter-related bloodstream infection appears much lower than that of secondary bloodstream infections [36].

However, there were some limitations in the present study. First, it was a retrospective study, and as a result, the patient characteristics, co-morbidities, and some other information were obtained based on the review of patient records rather than an interview or clinical examination at the time of infection, which might lead to some important information or variable such as Glasgow coma scale score could not be obtained accurately. Second, the current study was performed from a single center and the number of patients was relatively small, though it has reviewed the record of SA-BSI over a 6year period in our hospital. In addition, our institution is well-known in the field of trauma treatment nationwide, there was a considerable number of patients with trauma and burn in the study, which might lead to selection bias. Thus, the results from the current study might not be suitable for other hospitals. Third, it is possible that some important confounding variables for polymicrobial SA-BSI were not included and analyzed, as its intrinsic shortcoming from retrospective study. Thus, a multicentric study with a large sample size is necessary to further investigate the risk factors of polymicrobial SA-BSI for better prevention.

\section{Conclusions}

Polymicrobial SA-BSI is not a few events among Staphylococcus aureus bacteremia, and Acinetobacter baumannii is the predominant co-existed species. Burn injury, blood transfusion, mechanical ventilation, the length of prior hospital stay, and pneumonia as a primary site of infection are independent risk factors for polymicrobial SA-BSI. In addition, patients with polymicrobial SA-BSI might have worse outcomes compared with monomicrobial SA-BSI, which might be attracted more attention by physicians in the future.

\section{Abbreviations \\ SA-BSI: Staphylococcus aureus bloodstream infections; CCl: Charlson \\ Comorbidity Index; OR: Odds ratio; Cl: Confidence interval; ICU: Intensive care unit; BSI: Bloodstream infections; CNS: Coagulase-negative Staphylococcus; \\ GPT: Glutamic-pyruvic transaminase; GOT: Glutamic-oxaloacetic transaminase; APACHE: Acute physiology and chronic health evaluation; SOFA: Sequential organ failure assessment; CDC: Centers for Disease Control and Prevention; IQR: Interquartile range; MRSA: Methicillin-resistant Staphylococcus aureus; CLSI: Clinical and Laboratory Standards Institute; A. baumannii: Acinetobacter baumannii; S. aureus: Staphylococcus aureus}

\section{Acknowledgements}

Not applicable.

\section{Informed consent}

Due to the retrospective nature of the study, the Ethics Committee determined that no patient consent was required.

\section{Authors' contributions}

All authors contributed to data analysis, drafting or revising the article, gave final approval of the version to be published, and agree to be accountable for all aspects of the work.

\section{Funding}

This work was supported in part by grants from the National Natural Science Foundation of China (No. 81971871, GS Zhang; No. 81901941, SF Zhang); and the Natural Science Foundation of Zhejiang Province (No. LY19H150007, GS Zhang).

\section{Availability of data and materials}

All data generated or analyzed during this study are included in this manuscript.

\section{Ethics approval and consent to participate}

The present study received human research ethics approval (No. 2019-194) from the Ethics Committee of the Second Affiliated Hospital, Zhejiang University School of Medicine. We make sure to keep patient data confidential and compliance with the Declaration of Helsinki.

Consent for publication

Not applicable.

\section{Competing interests}

The authors declare that they have no competing interests.

\section{Author details}

${ }^{1}$ Department of Critical Care Medicine, Second Affiliated Hospital, Zhejiang University School of Medicine, Hangzhou 310009, China. ${ }^{2}$ Department of Critical Care Medicine, Taizhou Municipal Hospital, Taizhou 318000, Zhejiang, China. ${ }^{3}$ Department of Cardiology, Second Affiliated Hospital, Zhejiang University School of Medicine, Hangzhou 310009, China. ${ }^{4}$ Department of Critical Care Medicine, Taizhou Enze Medical Center (Group) Enze Hospital, Taizhou 318050, Zhejiang, China. ${ }^{5}$ Department of Critical Care Medicine, Huzhou First People's Hospital, Huzhou 313000, Zhejiang, China. ${ }^{6} \mathrm{Clinical}$ Microbiology Laboratory, Second Affiliated Hospital, Zhejiang University School of Medicine, Hangzhou 310009, China.

Received: 22 February 2020 Accepted: 16 May 2020

Published online: 27 May 2020

\section{References}

1. Rodriguez-Creixems M, Alcala L, Munoz P, Cercenado E, Vicente T, Bouza E. Bloodstream infections: evolution and trends in the microbiology workload, incidence, and etiology, 1985-2006. Medicine. 2008;87(4):234-49. 
2. Wisplinghoff $H$, Bischoff $T$, Tallent $S M$, Seifert $H$, Wenzel RP, Edmond MB. Nosocomial bloodstream infections in US hospitals: analysis of 24,179 cases from a prospective nationwide surveillance study. Clin Infect Dis. 2004;39(3): 309-17.

3. Uslan DZ, Crane SJ, Steckelberg JM, Cockerill FR 3rd, St Sauver JL, Wilson WR, et al. Age- and sex-associated trends in bloodstream infection: a population-based study in Olmsted County, Minnesota. Arch Intern Med. 2007:167(8):834-9.

4. Laupland KB, Gregson DB, Zygun DA, Doig CJ, Mortis G, Church DL. Severe bloodstream infections: a population-based assessment. Crit Care Med. 2004;32(4):992-7.

5. Weinstein MP, Towns ML, Quartey SM, Mirrett S, Reimer LG, Parmigiani G, et al. The clinical significance of positive blood cultures in the 1990s: a prospective comprehensive evaluation of the microbiology, epidemiology, and outcome of bacteremia and fungemia in adults. Clin Infect Dis. 1997; 24(4):584-602

6. Lin JN, Lai CH, Chen YH, Chang LL, Lu PL, Tsai SS, et al. Characteristics and outcomes of polymicrobial bloodstream infections in the emergency department: a matched case-control study. Acad Emerg Med. 2010;17(10): 1072-9

7. Weinstein MP, Reller LB, Murphy JR. Clinical importance of polymicrobial bacteremia. Diagn Microbiol Infect Dis. 1986;5(3):185-96.

8. Pavlaki M, Poulakou G, Drimousis P, Adamis G, Apostolidou E, Gatselis NK, et al. Polymicrobial bloodstream infections: epidemiology and impact on mortality. J Glob Antimicrob Resist. 2013;1(4):207-12.

9. Sancho S, Artero A, Zaragoza R, Camarena JJ, Gonzalez R, Nogueira JM. Impact of nosocomial polymicrobial bloodstream infections on the outcome in critically ill patients. Eur J Clin Microbiol Infect Dis. 2012;31(8): 1791-6.

10. Park SY, Park KH, Bang KM, Chong YP, Kim SH, Lee SO, et al. Clinical significance and outcome of polymicrobial Staphylococcus aureus bacteremia. J Inf Secur. 2012;65(2):119-27.

11. Khatib R, Sharma M, Johnson LB, Riederer K, Briski L. Polymicrobial Staphylococcus aureus bacteremia: frequency, distinguishing characteristics and outcome. Diagn Microbiol Infect Dis. 2016;86(3):311-5.

12. Bloodstream Infection (BSI) Events. Centers for Disease Control and Prevention. https://www.cdc.gov/nhsn/PDFs/pscManual/4PSC_ CLABScurrent.pdf. Accessed 20 May 2020.

13. Billington EO, Phang SH, Gregson DB, Pitout JD, Ross T, Church DL, et al Incidence, risk factors, and outcomes for Enterococcus spp. blood stream infections: a population-based study. Int J Infect Dis. 2014;26:76-82.

14. Garner JS, Jarvis WR, Emori TG, Horan TC, Hughes JM. CDC definitions for nosocomial infections, 1988. Am J Infect Control. 1988;16(3):128-40.

15. Li JS, Sexton DJ, Mick N, Nettles R, Fowler VG Jr, Ryan T, et al. Proposed modifications to the Duke criteria for the diagnosis of infective endocarditis. Clin Infect Dis. 2000;30(4):633-8.

16. Lodise TP, McKinnon PS, Swiderski L, Rybak MJ. Outcomes analysis of delayed antibiotic treatment for hospital-acquired Staphylococcus aureus bacteremia. Clin Infect Dis. 2003;36(11):1418-23.

17. Singer M, Deutschman CS, Seymour CW, Shankar-Hari M, Annane D, Bauer $M$, et al. The third international consensus definitions for Sepsis and septic shock (Sepsis-3). Jama. 2016;315(8):801-10.

18. Kiani D, Quinn EL, Burch KH, Madhavan T, Saravolatz LD, Neblett TR. The increasing importance of polymicrobial bacteremia. Jama. 1979;242(10): 1044-7.

19. Rello J, Quintana E, Mirelis B, Gurgui M, Net A, Prats G. Polymicrobial bacteremia in critically ill patients. Intensive Care Med. 1993;19(1):22-5.

20. Reuben AG, Musher DM, Hamill RJ, Broucke I. Polymicrobial bacteremia: clinical and microbiologic patterns. Rev Infect Dis. 1989;11(2):161-83.

21. Zheng C, Cai J, Liu H, Zhang S, Zhong L, Xuan N, et al. Clinical characteristics and risk factors in mixed-Enterococcal bloodstream infections. Infect Drug Resist. 2019;12:3397-407.

22. Zorgani A, Franka RA, Zaidi MM, Alshweref UM, Elgmati M. Trends in nosocomial bloodstream infections in a burn intensive care unit: an eightyear survey. Ann Burns Fire Disasters. 2010;23(2):88-94.

23. Church D, Elsayed S, Reid O, Winston B, Lindsay R. Burn wound infections. Clin Microbiol Rev. 2006;19(2):403-34

24. Santucci SG, Gobara S, Santos CR, Fontana C, Levin AS. Infections in a burn intensive care unit: experience of seven years. J Hosp Infect. 2003;53(1):6-13.
25. Fitzwater J, Purdue GF, Hunt JL, O'Keefe GE. The risk factors and time course of sepsis and organ dysfunction after burn trauma. J Trauma. 2003;54(5): 959-66.

26. Juffermans NP, Prins DJ, Vlaar AP, Nieuwland R, Binnekade JM. Transfusionrelated risk of secondary bacterial infections in sepsis patients: a retrospective cohort study. Shock. 2011;35(4):355-9.

27. Nacionales DC, Cuenca AG, Ungaro R, Gentile LF, Joiner D, Satoh M, et al. The acute immunological response to blood transfusion is influenced by polymicrobial sepsis. Shock. 2012;38(6):598-606.

28. Elting LS, Bodey GP, Fainstein V. Polymicrobial septicemia in the cancer patient. Medicine. 1986;65(4):218-25.

29. Mariya Joseph N, Sistla S, Kumar Dutta T, Shankar Badhe A, Rasitha D, Chandra PS. Outcome of ventilator-associated pneumonia: impact of antibiotic therapy and other factors. Australas Med J. 2012;5(2):135-40

30. Hess DR. Approaches to conventional mechanical ventilation of the patient with acute respiratory distress syndrome. Respir Care. 2011;56(10):1555-72.

31. Fagon JY, Chastre J, Hance AJ, Montravers P, Novara A, Gibert C. Nosocomial pneumonia in ventilated patients: a cohort study evaluating attributable mortality and hospital stay. Am J Med. 1993;94(3):281-8.

32. Garcia-Garmendia JL, Ortiz-Leyba C, Garnacho-Montero J, Jimenez-Jimenez FJ, Perez-Paredes C, Barrero-Almodovar AE, et al. Risk factors for Acinetobacter baumannii nosocomial bacteremia in critically ill patients: a cohort study. Clin Infect Dis. 2001;33(7):939-46.

33. Pan S, Huang $X$, Wang $Y$, Li L, Zhao C, Yao Z, et al. Efficacy of intravenous plus intrathecal/intracerebral ventricle injection of polymyxin $B$ for postneurosurgical intracranial infections due to MDR/XDR Acinectobacter baumannii: a retrospective cohort study. Antimicrob Resist Infect Control. 2018:7:8.

34. Ibrahim EH, Sherman G, Ward S, Fraser VJ, Kollef MH. The influence of inadequate antimicrobial treatment of bloodstream infections on patient outcomes in the ICU setting. Chest. 2000;118(1):146-55.

35. Cosgrove SE, Sakoulas G, Perencevich EN, Schwaber MJ, Karchmer AW, Carmeli Y. Comparison of mortality associated with methicillin-resistant and methicillin-susceptible Staphylococcus aureus bacteremia: a meta-analysis. Clin Infect Dis. 2003;36(1):53-9.

36. Renaud B, Brun-Buisson C. Outcomes of primary and catheter-related bacteremia. A cohort and case-control study in critically ill patients. Am J Respir Crit Care Med. 2001;163(7):1584-90.

\section{Publisher's Note}

Springer Nature remains neutral with regard to jurisdictional claims in published maps and institutional affiliations.

\section{Ready to submit your research? Choose BMC and benefit from:}

- fast, convenient online submission

- thorough peer review by experienced researchers in your field

- rapid publication on acceptance

- support for research data, including large and complex data types

- gold Open Access which fosters wider collaboration and increased citations

- maximum visibility for your research: over $100 \mathrm{M}$ website views per year

At BMC, research is always in progress.

Learn more biomedcentral.com/submissions 